Article

\title{
Optimization of a Power Line Communication System to Manage Electric Vehicle Charging Stations in a Smart Grid
}

\author{
Sara Carcangiu $(\mathbb{D}$, Alessandra Fanni $*(\mathbb{D})$ and Augusto Montisci $(\mathbb{}$ \\ Department of Electrical and Electronic Engineering, University of Cagliari, 09123 Cagliari, Italy; \\ s.carcangiu@diee.unica.it (S.C.); amontisci@diee.unica.it (A.M.) \\ * Correspondence: fanni@diee.unica.it; Tel.: +39-320-437-2966
}

Received: 26 February 2019; Accepted: 1 May 2019; Published: 9 May 2019

check for updates

\begin{abstract}
In this paper, a procedure is proposed to design a power line communication (PLC) system to perform the digital transmission in a distributed energy storage system consisting of fleets of electric cars. PLC uses existing power cables or wires as data communication multicarrier channels. For each vehicle, the information to be transmitted can be, for example: the models of the batteries, the level of the charge state, and the schedule of charging/discharging. Orthogonal frequency division multiplexing modulation (OFDM) is used for the bit loading, whose parameters are optimized to find the best compromise between the communication conflicting objectives of minimizing the signal power, maximizing the bit rate, and minimizing the bit error rate. The off-line design is modeled as a multi-objective optimization problem, whose solution supplies a set of Pareto optimal solutions. At the same time, as many charging stations share part of the transmission line, the optimization problem includes also the assignment of the sub-carriers to the single charging stations. Each connection between the control node and a charging station has its own frequency response and is affected by a noise spectrum. In this paper, a procedure is presented, called Chimera, which allows one to solve the multi-objective optimization problem with respect to a unique frequency response, representing the whole set of lines connecting each charging station with the central node. Among the provided Pareto solutions, the designer will make the final decision based on the control system requirements and/or the hardware constraints.
\end{abstract}

Keywords: power line communication (PLC); energy storage management; vehicle to grid (V2G); smart grid; multi-objective optimization

\section{Introduction}

Traditional power grids have been coupled with communication networks today, leading to the so-called smart grids. A smart grid enables information flows among various components of the grid, ranging from power plants to distributed energy resources, and from local utilities to residential and commercial customers. The purpose is to better monitor and control power generation and consumption. In smart grids, renewable sources, such as wind and solar energy, vary with weather and daylight conditions, so that an energy storage system is required to accumulate spare energy and to feed it back into the grid when required.

In smart grids, electric vehicles have an impact on energy storage through vehicle-to-grid (V2G) technologies [1], in which the electric vehicles (and even hybrids) can be seen as a distributed network of batteries that can store power at off-peak times and help power on the grid when demand peaks. Hence, V2G is useful to provide energy when demand shifts and to reduce electricity costs, to supply energy to energy markets, and to increase the use of localized renewables.

Each vehicle must have some requirements: 
1. A connection to the grid for electrical energy flow;

2. A control or logical connection necessary for communication with the grid operator;

3. Controls and metering onboard the vehicle; and

4. There must be an agreement between the owner of the battery and the grid operator that electricity can be put into or drawn from the battery.

Thus, it is important to have an automated and standardized exchange of information between the vehicles and the grid. In this regard, different protocols for the communication are used [2,3]: ISO/IEC 15118 concerns the communication between an electric vehicle and the charging spot whereas the IEC 61850 is related to the communication between the charging spot and the energy provider (Figure 1).

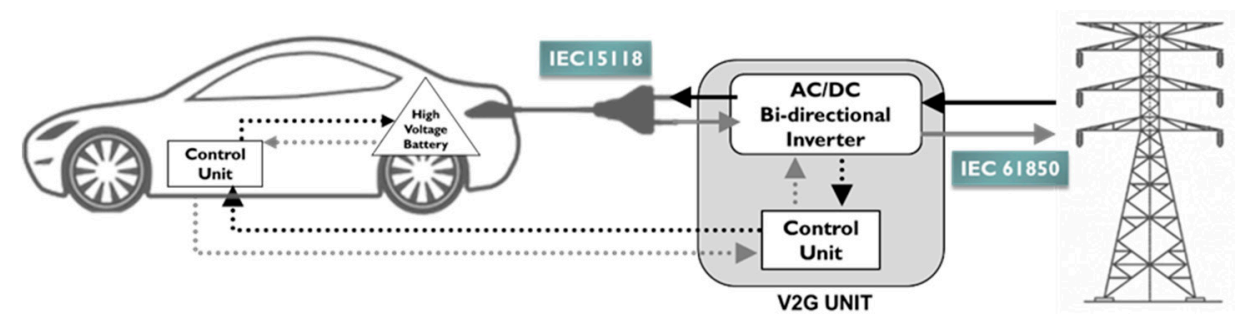

Figure 1. Vehicle-to-grid system.

A heterogeneous set of network technologies can support the smart grid communications ranging from wireless to wired solutions. Among the latter, PLCs have been deployed outdoor for last mile communications and indoor for home area networks [4]. A wide range of PLC technologies are available for different applications. Ultra-narrowband, operating at a very low data rate (100 bps) in the low-frequency band, is used in particular for load control. Narrowband PLC (NB PLC), operating in the $3-500 \mathrm{kHz}$ band to deliver a few hundred kbps, has been used for last mile communications over MV and LV lines. In order to transmit data over a narrowband PLC network, different specifications are used. The most used standards are PRIME, developed by the PRIME Alliance [5], and G3, powered by Maxim company [6]. Both the standards provide source coding techniques to correct as many errors as possible at the receiver side due to the severe channel disturbances. In the range $1.8-86 \mathrm{MHz}$ there is the broadband PLC (BB PLC), which is mainly used for home area networks and can provide several hundred Mbps. The typical examples of broadband PLCs conform to the standards IEEE 1901 [7], HomePlug [8], and ITU-T G.hn [9].

Both the NB-PLC and BB-PLC could be used in the smart grid applications, both in low voltage (LV) and medium voltage (MV) networks, with pros and cons [10,11]. NB-PLC are suitable for smart grid applications where a low-data rate is required, whereas BB-PLC solutions offer higher flexibility and a better trade-off between data rate, latency, robustness and energy efficiency [11]. In [12,13], the authors clarify the role of PLC technology for smart grid applications.

In this paper, to have the maximum flexibility, the BB-PLC technology is used. Specifically, in the V2G application, the most competitive advantage of using PLC is that no installation of an additional communication grid is required since the charging stations must be reached by the existing power grid to be fed. Moreover, the use of dedicated modulation techniques, such as OFDM (orthogonal frequency division multiplexing), allows for a broadband transmission, hence, PLC is competitive in more than in economic terms. Some disadvantages have to be taken into consideration: power lines do not necessarily provide a sure support, due to the presence of different elements; the attenuation of the data could be a problem; the electrical noise on the line limits the speed of the data transmission.

Designing the PLC system for the V2G needs a multi-objective optimization where some conflicting objectives should be considered: the communication capacity, the total transmission power, and the communication error probability. The multi-objective optimization process provides a set of equivalent solutions from which the designer makes the final choice, introducing further fitting criteria $[14,15]$. 
The optimization problem must be formalized depending on the modulation technique. Usually, to better exploit the available transmission band, the OFDM [8] system is assumed, which consists of splitting the transmission band into mutually orthogonal sub-carriers. Each one is used as a sub-carrier where the signal can be considered as if it were the only one in the channel. Every sub-carrier is loaded with a bit rate $(B R)$ depending on the specific signal-to-noise ratio (SNR). OFDM is a modulation scheme commonly adopted in several application domains, such as mobile phones, digital TV and radio, and XDSL, each one having specific features and requirements. In particular, in the application presented in the present paper, different transmitters share the same channel, therefore, each sub-carrier must be assigned exclusively to one transmitter.

The optimization of the modulation scheme works by allocating the bits to the sub-carriers, which is referred to as bit-loading [8] problem, and which depends on the properties of the channel and the requirements of the transmission. In the present paper, the optimal design of the modulation system in PLCs is formalized as a multi-objective optimization problem with the following three conflicting objectives: the $B R$, to be maximized; the bit error rate $(B E R)$ and the interference on adjacent equipment, to be minimized [16-18]. The interference mostly depends on the power of the modulated signal and on its evolution in the time.

The problem faced has its own specificity, since the transmissions of the charging stations travel through common branches of the grid. Consequently, they must share the same frequency band. However, each channel has its own frequency response and power spectral density of noise. Hence, the optimal solution of the design problem should consider all the channels simultaneously. Other applicative domains have the same specificity, for example, cellular phones or digital audio broadcasting (DAB) networks.

In the proposed method, the OFDM symbols, to be sent by all the transmitters, are merged into one, which simultaneously travels in all the channels. A fictitious frequency response, called Chimera, is created which allows to perform the multi-channel optimization of the OFDM modulation scheme as if all the transmissions should be sent through a unique channel (named here Chimera channel). To define the Chimera frequency response an integer quadratic optimization problem is formalized whose aim is to assign band resources to the charging stations connected to the same feeder, depending on the number of supplied plugs, maximizing, at the same time, the overall capacity of the transmission.

The rest of the paper is organized as follows. In Section 2, the problem at hand is outlined. In Section 3, the proposed Chimera algorithm is described. Section 4 reports the formalization of the multi-objective problem aiming to off-line design the PLC system. Results of the optimization procedure on a case study are reported in Section 5. Finally, in Section 6 some conclusions are given.

\section{V2G Communication System}

The present paper aims to optimally design a PLC system that allows to manage a distributed energy storage system integrated with a smart grid. The storage is composed of a number of electric vehicle batteries connected to prefixed charging stations. It is assumed that one modem is installed at the interface between the charging station and the smart grid. Therefore, the lines connecting the charging station to the plugs are not considered in the design problem.

To prove the validity of the method, a simple feeder network is considered, which is a part of a wider network already considered in [19]; this does not invalidate the generality of the results, as the method is applied separately for each single feeder. Moreover, frequency responses and noise spectra are obtained from simulations rather than measurements because the method can be demonstrated regardless of the actual data. Network Simulator 3 (NS-3) [20], which is a free and open source discrete event network simulator, has been used. Figure 2 shows the topology of the case study, with a number of low voltage nodes, $3 \mathrm{~kW}$ loads, and four charging stations (checkered squares in Figure 2) supplied by a medium voltage/low voltage substation with $20 \mathrm{kV} / 400 \mathrm{~V}, 1 \mathrm{MVA}$ transformer. All the lines are three-phase underground commercial cables, whose datasheets are included in the database of the simulator. A phase-neutral cable, among all those available, has been randomly chosen to be the PLC 
channel. The lengths of the lines are reported in Figure 2. Each charging station supplies a variable number of charging plugs and it is connected to the control center through the smart grid. As each charging station is connected to the control center by a different path, each one is associated to a different frequency response.

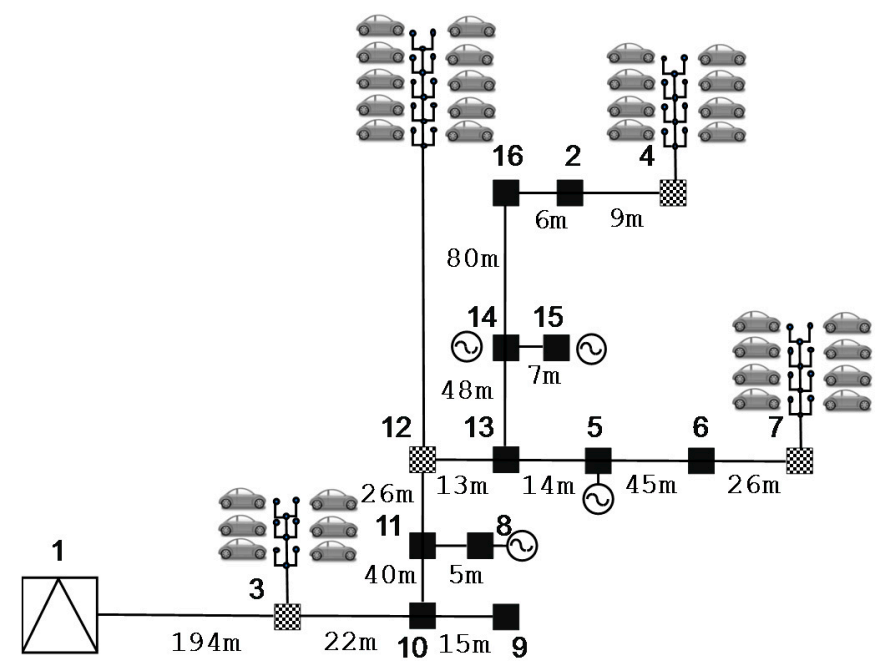

Figure 2. The case study: node 1 is the substation, the charging stations are the checkered nodes $3,4,7$, and 12 , whereas the black nodes are the low voltage $3 \mathrm{~kW}$ loads.

As known, the PLC channels are not time invariant due to load connection/disconnection [21]. For this reason, different scenarios have been simulated corresponding to different configurations of loads and active charging plugs and the lower envelop of the frequency responses has been assumed. In Figure 3, the power spectral densities (PSDs) of the signal as a function of the frequency in the nodes corresponding to the charging stations are shown. The frequency responses have been determined by feeding the channel with a white Gaussian noise and by measuring the spectrum of the signal at the receiver side. As a simplifying assumption, only the transmission from the charging station to the control center has been considered, without losing generality. Nonetheless, for each channel, two frequency responses for bi-directional transmission should be assessed, and a separate sub-band should be allocated to each. Thus, transmissions wouldn't need to be synchronized. Since the attenuation affects the performance of the PLC network, increasing with frequency and distance, longer LV lines must use frequencies in lower bands to guarantee a minimum performance.

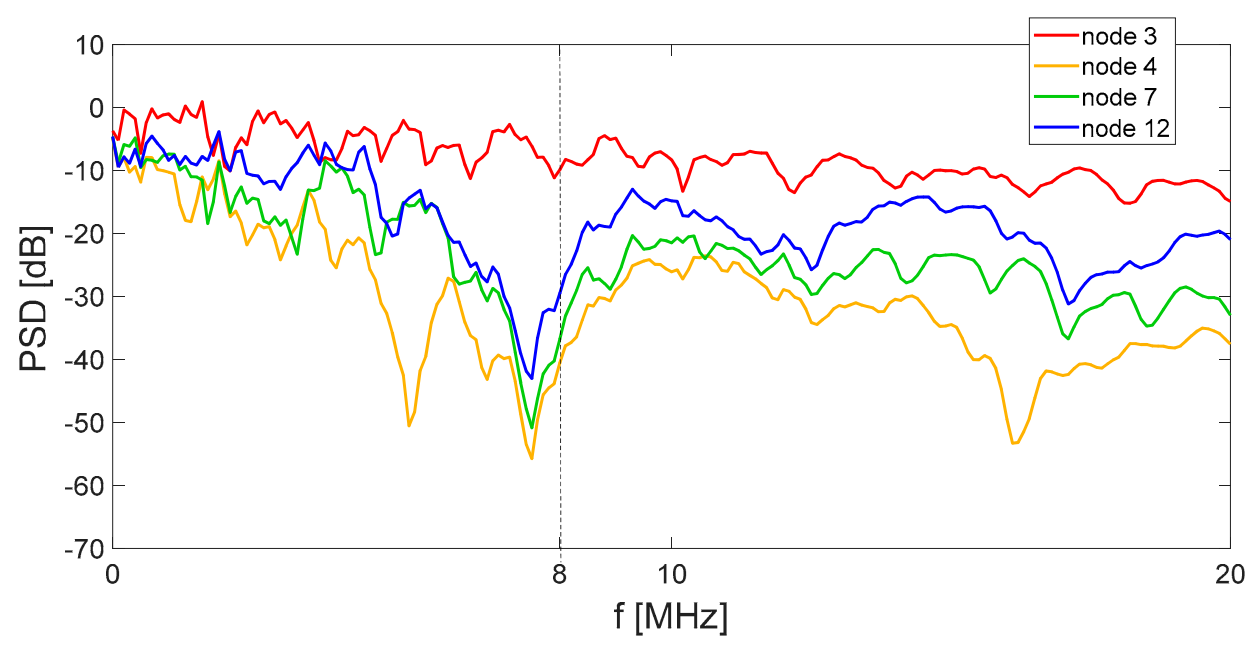

Figure 3. PSD of charging stations \#3, \#4,\#7, and \#12 connected to the control node 1. 
Depending on the occupation level of the channel, different information can be exchanged between the charging stations and the control system. Note that, because all the charging stations are connected to the same feeder, any transmission reaches all the nodes. However, as each sub-carrier is assigned exclusively to a specific node, each receiver is able to retrieve the transmission addressed to it.

Each node is connected to the control node through a power line, whose frequency response depends on the cable-laying and the length. For this reason, the optimal bit-loading should consider all the different frequency responses and all the possible states of the network. In order to reduce the design optimization complexity, a Chimera frequency response is built starting from the frequency responses of the nodes (the charging stations) connected to the considered feeder.

\section{Chimera Algorithm}

One of the most significant shortcomings of PLC is the frequency response of the transmission channel, as it has an irregular gain diagram, with deep notches, and an irregular phase diagram. OFDM technology allows mitigation of such shortcomings [22].

As the charging stations served by one feeder share the same physical channel that connects them to the control node, each frequency band has to be assigned exclusively to one charging station. The optimization of the OFDM modulation should take into account the different frequency responses of all the channels. In this paper, an algorithm, called Chimera, has been proposed to optimize the allocation of sub-carriers to the nodes served by the same feeder. The output of the algorithm is a single frequency response (Chimera), obtained by combining the entire set of frequency responses of the charging stations. At the same time, the set of transmissions of all nodes is merged into a single bit stream, which is assumed to travel through the Chimera channel.

The bit stream is first subdivided into frames of assigned numbers of bits, which have to be modulated. The frame, in turn, is subdivided into as many strings of bits (words) as the number of sub-carriers, and the length of each word is assigned according to the capacity of the sub-carrier where it will be loaded.

Depending on the single capacity, a constellation, defined in the complex plane, is associated with each sub-carrier. Each point of the constellation is associated to a string of bits, so that each possible word can be transmitted by sending the coordinates of the corresponding point. Therefore, the length of each word, i.e., the number of bits, is equal to the base-2 logarithm of the number of points in the constellation. Having a constellation with many points favors the bit rate, but, at the same time, this reduces the margin among near points, which affects the BER of receiving data. In this paper, only a limited number of design parameters are considered, such as the transmission band and its subdivision into sub-carriers, the allocation of bits to each sub-carrier (bit loading) and the maximum power of transmission. Further design parameters, such as zero-padding, cyclic prefix, time guard, and so on [22,23], have been considered here by assuming a proper margin for the duration of the single OFDM symbol.

Let $N$ be the number of nodes of the feeder that share the same transmission band, and $K$ the number of sub-carriers in which the transmission band is subdivided. Each node is associated with a specific frequency response (see Figure 3). It is assumed that each node supplies $V_{n}$ plugs, whose number can be different for different nodes. Moreover, it is assumed that the nodes communicate only with the central node, therefore, a number of frequency responses equal to the number of nodes have to be taken into account. The same subdivision in sub-bands is applied to all those frequency responses.

The capacity $C_{n k}$ of the $k$-th sub-carrier of the channel $n$ is given by the formula of Shannon-Hartley [24], which depends on the bandwidth $B$ and the signal-to-noise ratio (SNR):

$$
C_{n k}=B \cdot \log _{2}\left(1+S N R_{n k}\right)[b i t / s]
$$

The value in Equation (1) is the theoretical upper bound of $B R$ in the sub-carrier, but a lower value of $B R$ is assumed in order to maintain the $B E R$ within an acceptable threshold. 
The bandwidth is common to all the sub-carriers of all the frequency responses, therefore from here on we consider a normalized value of $B$. The total capacity of $n$th channel, corresponding to a specific node, is given by the sum of the capacities of all sub-carriers:

$$
C_{n}=\sum_{k=1}^{K} C_{n k}
$$

This expression can be calculated for each frequency response, and it assumes that all the sub-carriers are assigned to each node. An incidence matrix $M_{N x K}$ is used to represent the assignment of sub-carriers to the single nodes. The columns of $M$ correspond to the sub-carriers, whereas the rows correspond to the channels. In each row, each 1 identifies a sub-carrier assigned to the corresponding node. Each column can have at most one element equal to 1 and the others are equal to 0 . The columns with all zeros correspond to not assigned sub-carriers. By using matrix $M$, the capacity $\tilde{C}_{n}$ assigned to each node can be expressed as:

$$
\tilde{C}_{n}=\sum_{k=1}^{K} m_{n k} C_{n k}=\sum_{k=1}^{K} m_{n k} \log _{2}\left(1+S N R_{n k}\right)
$$

where $m_{n k}$ is the element of $M$ corresponding to the frequency response $n$ and the sub-carrier $k$. The aim of the procedure is to assign to each charging station a capacity proportional to the number of plugs it feeds. This rule can be expressed by the following statement:

$$
\frac{1}{V_{1}} \sum_{k=1}^{K} m_{1 k} C_{1 k}=\ldots=\frac{1}{V_{N}} \sum_{k=1}^{K} m_{N k} C_{N k}
$$

The allocation problem consists in defining the matrix $M$. As the unknowns are binary, the previous series of equations has not, in general, an exact solution, therefore the following objective function is defined, which has to be minimized:

$$
J=\sum_{n=1}^{N}\left(\frac{1}{V_{s}} \sum_{k=1}^{K} m_{s k} C_{s k}-\frac{1}{V_{n}} \sum_{k=1}^{K} m_{n k} C_{n k}\right)^{2}
$$

where $s$ identifies the channel with the lowest total capacity:

$$
s=\underset{n \in\{1, \ldots, N\}}{\operatorname{argmin}}\left(\frac{1}{V_{n}} \sum_{k=1}^{K} C_{n k}\right)
$$

The quadratic function (5) allows to obtain the most homogeneous distribution of capacities, but it does not guarantee the total capacity is maximized. For this reason, a further term is added to the objective function, which weighs the capacity assigned to the channel with the lowest total capacity, obtaining:

$$
J=\sum_{n=1}^{N}\left(\frac{1}{V_{s}} \sum_{k=1}^{K} m_{s k} C_{s k}-\frac{1}{V_{n}} \sum_{k=1}^{K} m_{n k} C_{n k}\right)^{2}-\alpha \sum_{k=1}^{K} m_{s k} C_{s k}
$$

where $\alpha$ is the weight coefficient; a small value favors the homogeneity among the channels, while a large value tends to increase the capacities assigned to all the channels. Hence, the best $\alpha$ is the one that maximizes the lowest allocated capacity.

In order to avoid both the trivial solution of null matrix $M$, and the same sub-carrier be assigned to different channels, the following constraint has to be stated:

$$
\sum_{n=1}^{N} m_{n k}=1 \quad \forall k=1, \ldots, K
$$

In case one sub-carrier is not suitable for the assignment, the corresponding column of the matrix $M$ has to be removed. 
Summarizing, the allocation problem can be defined as the following quadratic, binary minimization problem:

$$
\begin{gathered}
\min J \\
\text { s.t. } \\
\sum_{n=1}^{N} m_{n k}=1 \quad \forall k=1, \ldots, K \\
m_{n k} \in\{0 ; 1\}
\end{gathered}
$$

\section{Multi-Objective Optimization}

The demand of transmission is maximizing the Bit Rate (BR) (bit/s), which is equal to the number of bits per frame multiplied by the number of OFDM symbols per second. Two other conflicting objectives must be minimized at once. The first is the total power of the signal $P_{t o t}$, which is the sum of the powers of all the sub-carriers. The second conflicting objective is the error bit rate $(B E R)$, which depends on the noise spectrum: a probability density function can be associated to each point of the constellation centered in these points and the $B E R$, for a given point, will be equal to the probability that the distance between a received point and its reference position be greater than the semi-distance between two adjacent points. In this paper, Gaussian distribution is assumed, so that there is a direct relationship between $B E R$ and the least distance among the points of the constellation. Different assumptions on the statistical distribution of points around the reference position do not change the application of the proposed method.

Note that, by setting the maximum transmission power of each constellation $\left(P_{M A X}\right)$, its area is univocally assigned (area of the outer circle in Figure 4). Then, by setting the minimum distance between the points of the constellation (i.e., the $B E R$ ), also its number of points is determined, hence, also the $B R$.

Figure 4 refers to the 16-APSK scheme for a generic sub-carrier [25]. In the same figure, the shadow circles centered in the points of the constellation have a radius equal to 3 times the standard deviation $\sigma$ of the Gaussian distribution. The distance between two adjacent points is greater than $6 \sigma$.

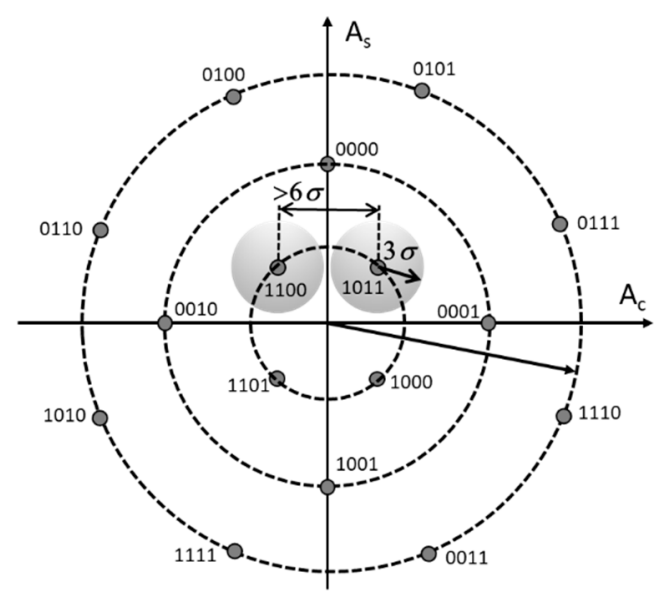

Figure 4. The 16-APSK constellation.

The requirements on the BER are particularly stringent, therefore, the APSK constellations [25] are adopted, which are increasingly considered in $5 \mathrm{G}$ mobile communications; for a given power, APSK gives the maximum distance between adjacent points. Without loss of generality, different constellation schemes can be assumed because the inter-dependence among power, $B R$ and $B E R$ holds valid.

The water-filling (WF) algorithm [26,27] is used to allocate the bits to the sub-carriers. Note that, WF can be used either to design the constellations or to dynamically allocate the bits to the sub-carriers. In this work, it has been used for the former purpose.

The multi-objective optimization of the bit loading consists in finding the solutions that reconcile the conflicting demands. In particular, a solution is said nondominated, or Pareto optimal, if none of 
the objective functions can be improved in value without worsening some of the others. The set of such solutions is called Pareto Front [14] and, in the problem at hand, it represents a discrete subspace of the surface of the feasible solutions. In fact, as $B R$ is a discrete variable, except for a discrete set of points in the $\left(P_{t o t}, B E R\right)$ plane, the increase of one or both of them does not correspond to an increase of $B R$.

The multi-objective optimization aims to find this set of Pareto solutions and then to present them to the designer who will choose among them $[14,15]$. In the problem at hand, the surface $B R=f\left(P_{t o t}, B E R\right)$ has been densely sampled, and the set of non-dominated points has been selected, which is assumed as the Pareto front.

\section{Results}

The optimization problem in (9) is solved with a genetic algorithm implemented in the Matlab environment [28]. A transmission band of $1-8 \mathrm{MHz}$ is assumed, because the SNR is unfavorable outside such interval. A width of $100 \mathrm{kHz}$ has been assigned to each sub-carrier, then 70 sub-carriers are available. In practice, the number of actually available sub-carriers could be much lower, because of the great attenuation of the power line and the presence of noise. In this work, for each channel, the power spectrum of the noise has been obtained with the same NS3 simulator, which is in good agreement with experimental results retrieved from the literature [21,29].

The number of variables to be optimized is equal to 280 (70 sub-carriers for four channels), and the initial population has been generated using a uniform random number generator in the range $\{0 ; 1\}$ After 28,000 iterations the algorithm ends producing the matrix $M$. This allows us to obtain a single frequency response for all the charging stations by multiplying $M$ with their frequency responses. The values of the genetic algorithm parameters are reported in Table 1. Figure 5 shows the obtained Chimera frequency response assumed to represent all the charging stations in Figure 2. The weight coefficient $\alpha$ has been set equal to 1 .

Table 1. Genetic algorithm parameters.

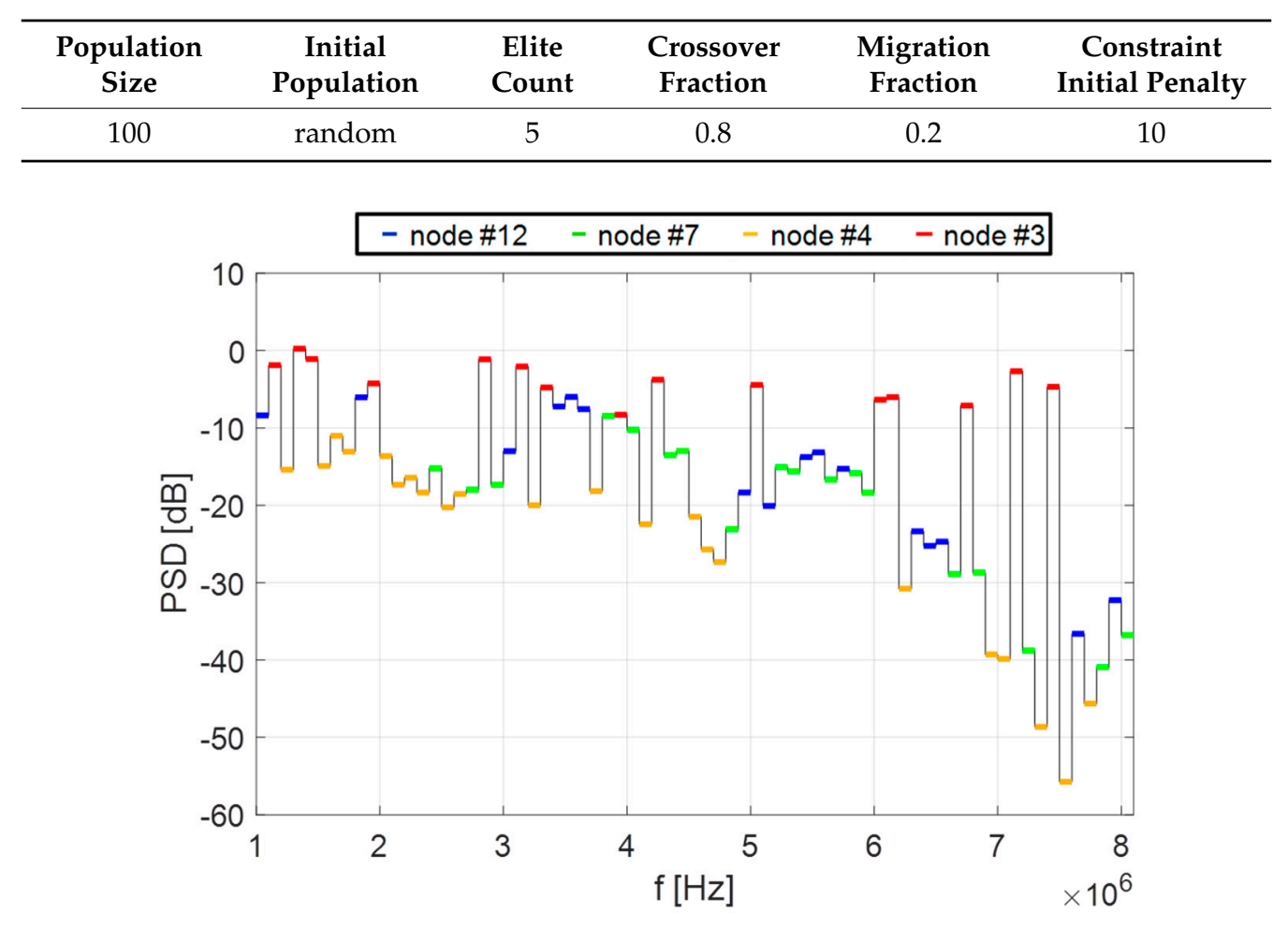

Figure 5. Power spectral density of the Chimera transmission channel: the different colors refer to the allocation of the 4 charging nodes to the 70 sub-carriers. 
To perform the offline optimization of $B R, B E R$ and $P_{t o t}$, the function $B R=f\left(P_{t o t}, B E R\right)$ is sampled in a regular $100 \times 100$ grid, within the interval of $1-10[\mathrm{~W}]$ of $P_{\text {tot }}$, and $10^{-5}-10^{-3}$ of $B E R$. The upper envelope of the noise PSDs of the four channels has been assumed as unique noise spectrum, as shown in Figure 6. Note that, given the Chimera frequency response and the noise, by applying the water filling algorithm, the power value for each sub-carrier is defined as a function of the total transmitting power $P_{\text {tot }}$. Hence, given the noise, the standard deviation $\sigma$ is known, then the minimal inter-distance between the constellation points is determined. In this way, the number of points of the constellation is calculated, by obtaining the number of bits associated to each point, then the length of bit frame is obtained, and, finally, the $B R$.

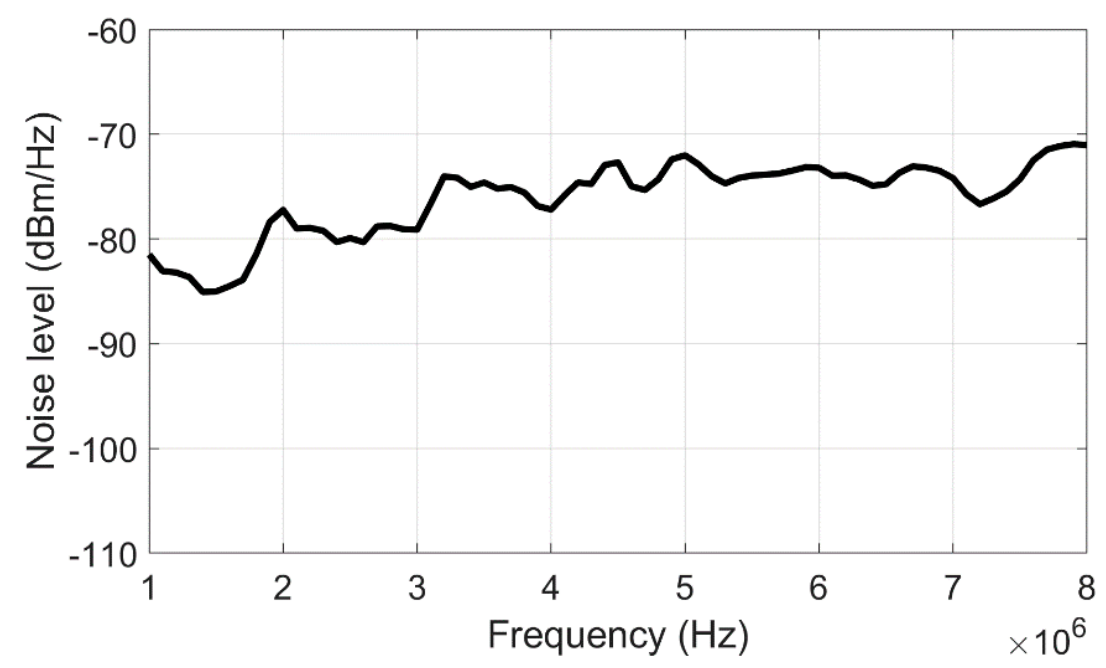

Figure 6. Noise power spectrum.

The sampled surface is shown in Figure 7. The non-dominated points are also represented by dots. The relationship between the three objectives allows us to obtain a $B R$ ranging from $24.3-60 \mathrm{Mb} / \mathrm{s}$.

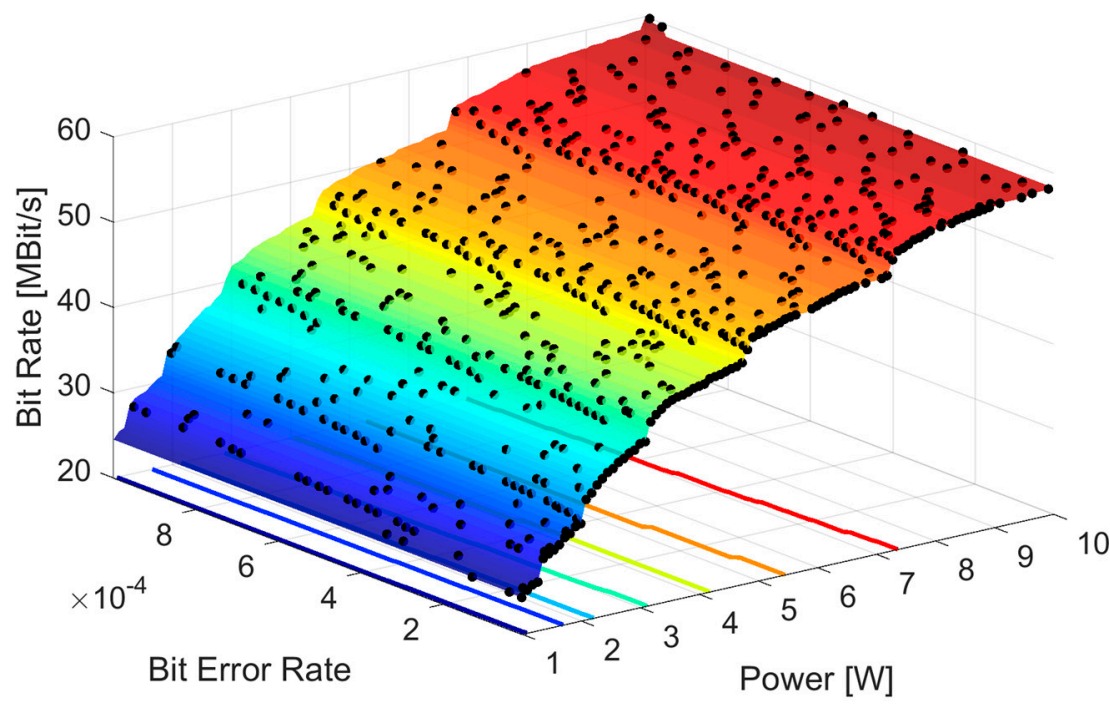

Figure 7. $B R$ as function of $B E R$ and $P_{t o t}$, and Pareto front (dots).

Figure 8 gives an equivalent representation of the Pareto front in the plane $\left(P_{t o t}, B E R\right)$. It is a contour plot of the front, and the points represent the Pareto-optimal solutions. As it can be noted, in order to have a minimal $B R$ at least a power of $1 \mathrm{~W}$ is needed. Moreover, limiting the available power to about $7 \mathrm{~W}$, a $B R$ of $52.1 \mathrm{Mb} / \mathrm{s}$ can be achieved, with a $B E R$ of the order of $10^{-5}$. If a higher $B R$ (e.g., $60 \mathrm{Mb} / \mathrm{s}$ ) must be achieved, the $B E R$ will strongly affect the transmission. 


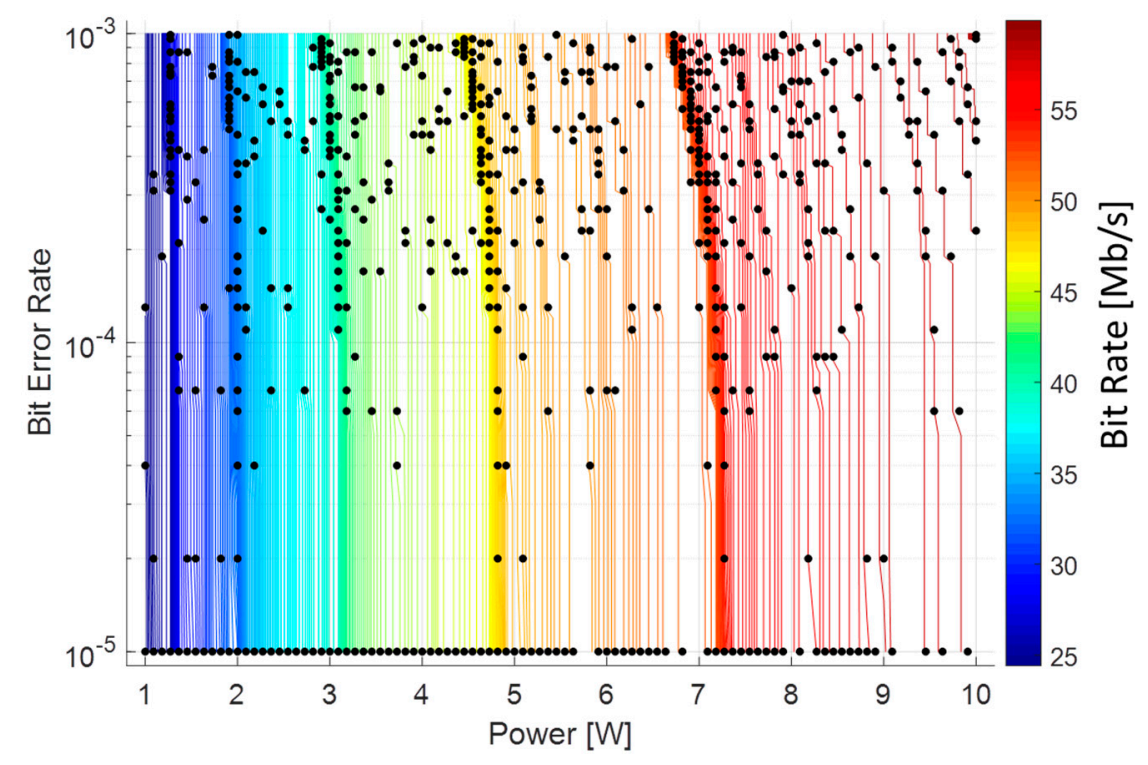

Figure 8. Two-dimensional representation of the Pareto front.

Figure 9 shows the Pareto optimal solutions corresponding to the minimal $B E R=10^{-5}$. Depending on the available total transmitting power, the plot returns the available $B R$ for each plug in kByte/s. As an example, with an available transmitting power of $5 \mathrm{~W}$, the $B R$ is equal to about $190 \mathrm{kByte} / \mathrm{s}$ for each of the 32 plugs in the four charging stations.

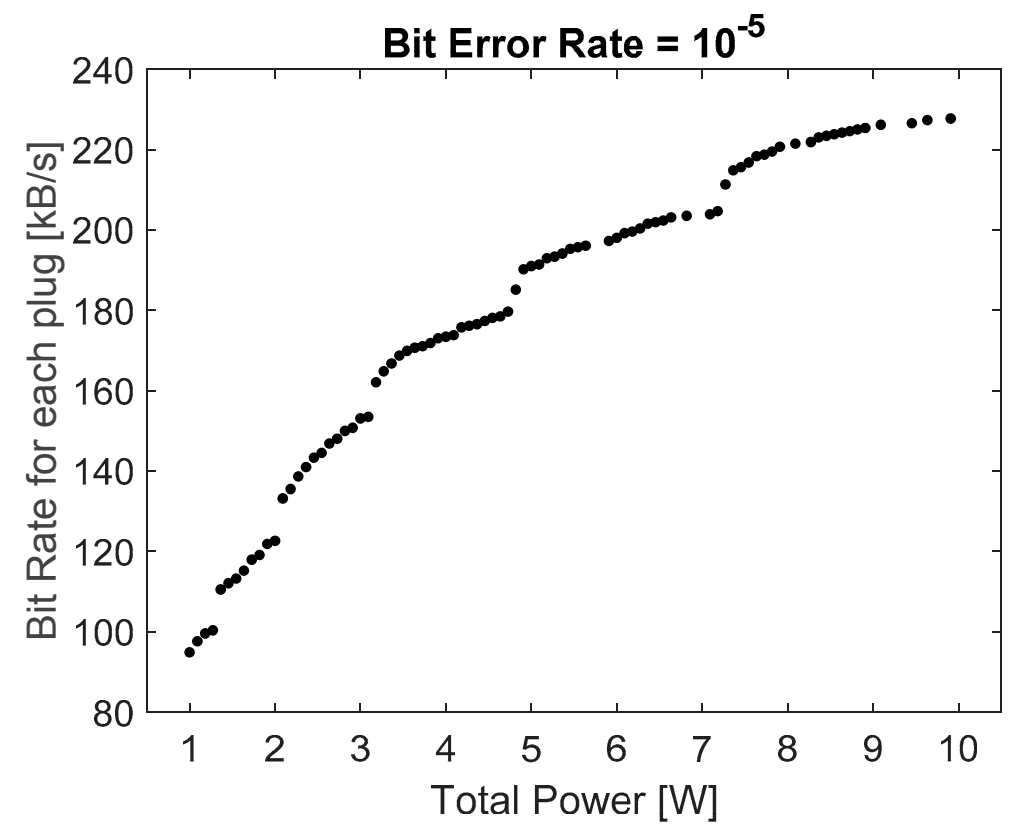

Figure 9. Pareto optimal solutions with a $B E R=10^{-5}$.

Finally, in Figure 10 the 70 APSK constellations corresponding to the Pareto point $(B R=190 \mathrm{kB} / \mathrm{s}$, $B E R=10^{-5}, P=5 \mathrm{~W}$ ) are shown. The colors refer to the different charging stations. As can be noted, the optimization procedure assigned different constellations to distinct sub-carriers. 


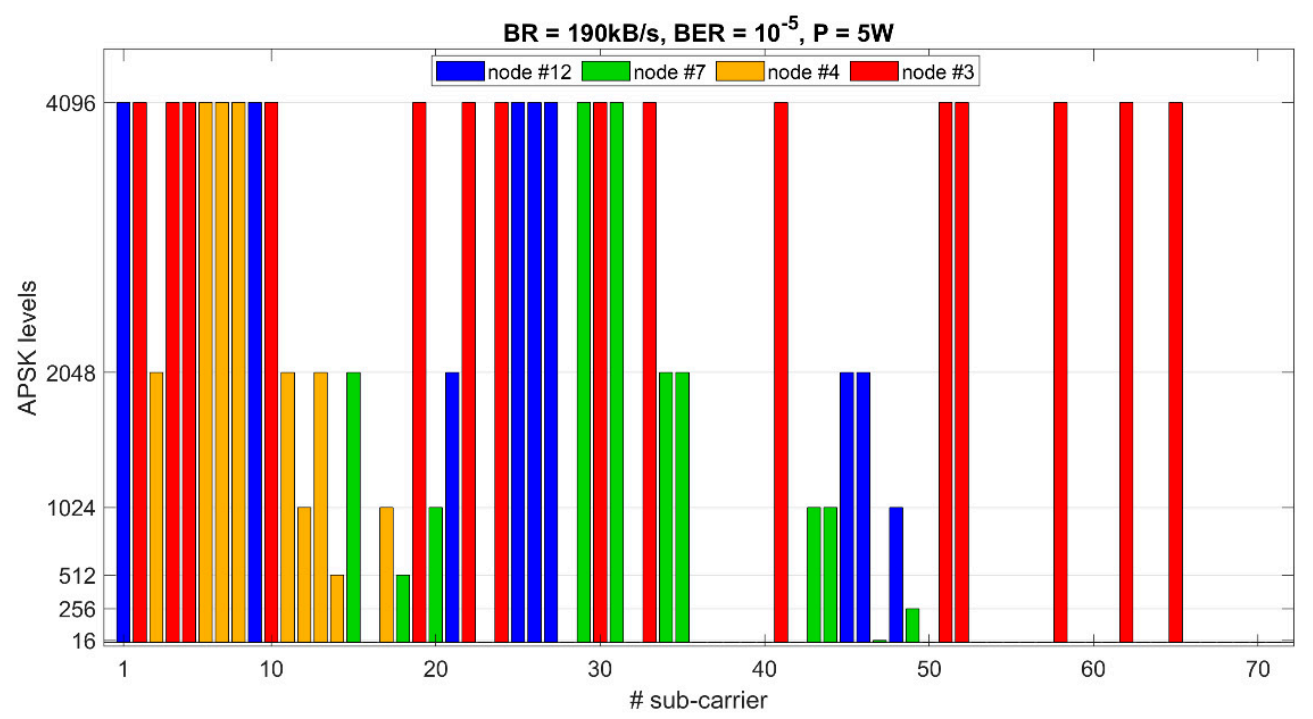

Figure 10. APSK OFDM constellations corresponding to the Pareto point $B R=190 \mathrm{kB} / \mathrm{s}, B E R=10^{-5}$, $P=5 \mathrm{~W}$.

\section{Discussion}

In a smart grid environment new challenges are posed to the power system operators by electric vehicles. Several studies have been proposed in the literature presenting control and optimization strategies for managing the charging/discharging of the electric vehicles' batteries [30]. However, the communication requirements still remain an open issue. In fact, different charging and discharging management strategies can be adopted ranging from a fully-centralized charge control decision, to distributed (or transactive), and to price control, which has limited communication requirements [31]. In the first case, the decisions are taken at the system level, hence it is generally accepted that it is the best control system in terms of, e.g., security of the power system. However, a more sophisticated communication infrastructure is needed that foresees bidirectional communication flow at the price of higher cost. Power line communication technology stands as a good candidate provided that its design is optimized. The design procedure presented in this paper, allows one to solve the bit-loading problem, finding a compromise among the conflicting objectives of minimal signal power and BER, while maximizing the $B R$. Among the Pareto-optimal solutions, provided by the optimization process, the designer can perform the definitive choice depending on the hardware and the communication requirements. Once this choice is made, the entire constellation system is designed.

Author Contributions: Conceptualization and analyses were done through the collaboration of all the authors. Software development was done by S.C. Data validation was done by A.M. A.F. wrote and reviewed the paper. All authors have read and approved the final manuscript.

Funding: This research has been funded by "Fondazione di Sardegna" under project "ODIS_Optimization of DIstributed systems in the Smart-city and smart-grid settings", CUP: F72F16003170002.

Conflicts of Interest: The authors declare no conflict of interest. The funders had no role in the design of the study; in the collection, analyses, or interpretation of data; in the writing of the manuscript; or in the decision to publish the results.

\section{References}

1. He, Y.; Bhavsar, P.; Chowdhury, M.; Li, Z. Optimizing the performance of vehicle-to-grid (V2G) enabled battery electric vehicles through a smart charge scheduling model. Int. J. Automot. Technol. 2015, 16, 827-837. [CrossRef]

2. Käbisch, S.; Schmitt, A.; Winter, M.; Heuer, J. Interconnections and communications of electric vehicles and smart grids. In Proceedings of the 1st IEEE International Conference on Smart Grid Communications (SmartGridComm), Gaithersburg, MD, USA, 4-6 October 2010; pp. 161-166. 
3. Hansch, K.; Pelzer, A.; Komarnicki, P.; Groning, S.; Schmutzler, J.; Wietfeld, C.; Heuer, J.; Muller, R. An ISO/IEC15118 conformance testing systemarchitecture. In Proceedings of the IEEE-PES General Meeting, Conference \& Exposition, National Harbor, MD, USA, 27-31 July 2014; pp. 1-5.

4. Lampe, L.A.; Tonello, M.; Swart, T.G. Power Line Communications: Principles, Standards and Applications from Multimedia to Smart Grid, 2nd ed.; John Wiley \& Sons: Hoboken, NJ, USA, 2016.

5. Berganza, I.; Sendin, A.; Arriola, J. PRIME: Powerline Intelligent Metering Evolution. In Proceedings of the CIRED Seminar 2008, Frankfurt, Germany, 23-24 June 2008.

6. The G3-PLC Alliance. Available online: http://www.g3-plc.com/ (accessed on 8 January 2019).

7. Medium Access Control and Physical Layer Specifications; IEEE Std. 1901-2010; IEEE Standard Association: New York, NY, USA, 30 December 2010.

8. Guerrini, E.; Dell' Amico, G.; Bisaglia, P.; Guerrieri, L. Bit-loading algorithms and SNR estimate for HomePlug AV. In Proceedings of the IEEE International Symposium on Power Line Communications and its Applications, ISPLC 2007, Pisa, Italy, 26-28 March 2007; pp. 77-82.

9. Unified High-Speed Wireline-Based Home Networking Transceivers-System Architecture and Physical Layer Specification. ITU-T Rec. G.9960. 2011. Available online: https://www.itu.int/rec/T-RECG.9960/en (accessed on 8 January 2019).

10. Tonello, A.M.; Versolatto, F.; Pittolo, A. In-home power line communication channel: Statistical characterization. IEEE Trans. Commun. 2014, 62, 2096-2106. [CrossRef]

11. Tonello, A.M.; Pittolo, A. Considerations on Narrowband and Broadband Power Line Communication for Smart Grids. In Proceedings of the IEEE Smart Grid Communications 2015, Miami, FL, USA, 2-5 November 2015.

12. Ferreira, H.C.; Lampe, L.; Newbury, J.; Swart, T.G. Power Line Communications: Theory and Applications for Narrowband and Broadband Communications over Power Lines; Wiley \& Sons: Hoboken, NJ, USA, 2010.

13. Galli, S.; Scaglione, A.; Wang, Z. For the Grid and Through the Grid: The Role of Power Line Communications in the Smart Grid. Proc. IEEE 2011, 99, 998-1027. [CrossRef]

14. Carcangiu, S.; Fanni, A.; Montisci, A. Multiobjective tabu search algorithms for optimal design of electromagnetic devices. IEEE Trans. Magn. 2008, 44, 970-973. [CrossRef]

15. Carcangiu, S.; Fanni, A.; Montisci, A. Multi objective optimization algorithm based on neural networks inversion. Lect. Notes Comput. Sci. 2009, 5517, 744-751. [CrossRef]

16. Carcangiu, S.; Montisci, A.; Usai, M. Bit Loading Optimization for Naval PLC Systems. In Proceedings of the IEEE International Symposium on Power Line Communications and its Applications, Udine, Italy, 3-6 April 2011; pp. 84-89.

17. Camplani, M.; Cannas, B.; Carcangiu, S.; Fanni, A.; Montisci, A.; Usai, M. Tabu-Search Procedure for PAPR Reduction in PLC Channels. In Proceedings of the IEEE International Symposium on Industrial Electronics, Bari, Italy, 4-7 July 2010; pp. 2979-2983.

18. Carcangiu, S.; Montisci, A. A Hybrid Analytical-Numerical Approach for PAPR Reduction in PLC Grids. In Proceedings of the 8th Mediterranean Conference on Power Generation, Transmission, Distribution and Energy Conversion, MEDPOWER 2012, Cagliari, Italy, 1-3 October 2012; pp. 1-5.

19. Carcangiu, S.; Celli, G.; Fanni, A.; Garau, M.; Montisci, A.; Pilo, F. Bit loading optimization for smart grid energy storage management. In Proceedings of the IEEE 3rd International Forum on Research and Technologies for Society and Industry, RTSI 2017, Modena, Italy, 11-13 September 2017. 8065946.

20. Aalamifar, F.; Schlögl, A.; Harris, D.; Lampe, L. Modelling power line communication using network simulator-3. In Proceedings of the IEEE Global Communications Conference (GLOBECOM), Atlanta, GA, USA, 9-13 October 2013; pp. 2969-2974.

21. Barmada, S.; Bellanti, L.; Raugi, M.; Tucci, M. Analysis of Power-Line Communication Channels in Ships. IEEE Trans. Veh. Technol. 2010, 59, 3161-3170. [CrossRef]

22. Ma, Y.H.; So, P.L.; Gunawan, E. Performance analysis of OFDM systems for broadband power line communications under impulsive noise and multipath effects. IEEE Trans. Power Deliv. 2005, 20, 674-682. [CrossRef]

23. Muquet, B.; Wang, Z.; Giannakis, G.B.; de Courville, M.; Duhamel, P. Cyclic prefixing or zero-padding for wireless multicarrier transmissions? IEEE Trans. Commun. 2002, 50, 2136-2148. [CrossRef]

24. Carlson, A.B. Communication Systems, 3rd ed.; McGraw-Hill: New York, NY, USA, 1986. 
25. Liu, Z.; Xie, Q.; Peng, K.; Yang, Z. APSK Constellation with Gray Mapping. IEEE Commun. Lett. 2011, 15, 1271-1273. [CrossRef]

26. Honda, S.; Umehara, D.; Hayasaki, T.; Denno, S.; Morikura, M. A fast bit loading algorithm synchronized with commercial power supply for in-home PLC systems. In Proceedings of the IEEE International Symposium on Power Line Communications and Its Applications, ISPLC 2008, Jeju City, Jeju Island, Korea, 2-4 April 2008; pp. 336-341.

27. Pérez Palomar, D.; Rodríguez Fonollosa, J. Practical Algorithms for a Family of Waterfilling Solutions. IEEE Trans. Signal Process. 2005, 53, 686-695. [CrossRef]

28. MathWorks. Available online: https://www.mathworks.com/help/gads/ga.html (accessed on 8 January 2019).

29. Di Bert, L.; Caldera, P.; Schwingshackl, D.; Tonello, A.M. On noise modeling for power line communications. In Proceedings of the IEEE International Symposium on Power Line Communications and Its Applications, ISPLC 2011, Udine, Italy, 3-6 April 2011; pp. 283-288.

30. Samy Faddel, S.; Al-Awami, A.T.; Mohammed, O.A. Charge Control and Operation of Electric Vehicles in Power Grids: A Review. Energies 2018, 11, 701. [CrossRef]

31. Hu, J.; Morais, H.; Sousa, T.; Lind, M. Electric vehicle fleet management in smart grids: A review of services, optimization and control aspects. Renew. Sustain. Energy Rev. 2016, 56, 1207-1226. [CrossRef]

(C) 2019 by the authors. Licensee MDPI, Basel, Switzerland. This article is an open access article distributed under the terms and conditions of the Creative Commons Attribution (CC BY) license (http://creativecommons.org/licenses/by/4.0/). 